\title{
Industries et Services ruraux: Supports de la Transformation Rurale dans le contexte d'une Agriculture en dégradation continue : Evidence du Sénégal
}

\author{
Amadou Tandjigora \\ Faculté des Sciences Economiques et de Gestion (FASEG) \\ Université Cheikh Anta Diop de Dakar (UCAD)
}

\begin{abstract}
Résumé :
L'objectif de cet article est de trouver une alternative à la faiblesse de revenus de la plupart des ménages ruraux du Sénégal dont la principale cause est la dépendance d'une agriculture à faible productivité. La méthodologie que nous avons utilisée à cet effet est celle d'une étude comparative entre les revenus provenant de l'agriculture et ceux provenant des petites industries et services ruraux (services, commerce, autres services). Pour ce faire, le modèle logit ordonné a été utilisé pour effectuer les estimations. Les résultats obtenus ont montré que les revenus provenant de ces différentes activités non agricoles sont largement supérieurs à ceux pouvant être générés par l'activité économique principale qu'est l'agriculture. Là où l'agriculture rapporte 1 Franc CFA à son pratiquant, le service rapporterait au même moment 12 Francs CFA à son pratiquant, l'industrie quant à elle, rapporterait 3,87 Francs CFA, le commerce génèrerait 1,85 Francs CFA tandis que les autres services fourniraient 4,83 Francs CFA à son pratiquant. Les ménages, surtout ceux les plus vulnérables à savoir les femmes, les jeunes et les pauvres sans terres ou avec juste de petites parcelles insuffisantes pour satisfaire leurs besoins essentiels, doivent entreprendre au moins une activité de ces différents secteurs (industrie et services) comme principal emploi. Cette logique ou alternative de substitution s'inscrit dans un processus de transformation rurale constaté dans nombre de pays en développement dont l'économie dépend plus de l'agriculture qu'à d'autres secteurs d'activité. L'article fournit des résultats concrets pouvant permettre aux ménages ruraux de faire face à une éventuelle décapitalisation causée le plus souvent par des conditions climatiques défavorables.
\end{abstract}

Mots clés : Transformation rurale, Industrie, Services, Logit ordonné, Sénégal.

DOI: $10.7176 / \mathrm{JESD} / 10-2-05$

\begin{abstract}
:
The purpose of this article is to find an alternative to the low income of most rural households in Senegal whose main cause is the dependence of low agriculture productivity. The methodology used for this purpose is a comparative study between income from agriculture and that from small industries and rural services (services, trade, other services). The ordered logit model was used to make the estimates. The results obtained show that incomes from these different non-agricultural activities are much higher than that generated by the main economic activity that is agriculture. Where the agriculture pays 1 CFA Franc to his practitioner, the service would bring at the same time 12 CFA Francs to his practitioner, the industry meanwhile, would bring back 3,87 CFA Francs, the trade would generate 1,85 CFA Francs while the other services would provide 4.83 CFA Francs. Households, especially the most vulnerable, namely women, the young and the landless poor or with just small parcels insufficient to meet their basic needs, must undertake at least one activity from these different sectors (industry and services) as the main employment. This logic or substitution alternative is part of a rural transformation process seen in many developing countries whose economy depends more on agriculture than on other sectors of activity. The article provides concrete results that can enable rural households to cope with a possible decapitalization caused mostly by adverse weather conditions.
\end{abstract}

\section{INTRODUCTION}

En raison du déficit quantitatif (chronique) de la pluviométrie, de l'aridité des sols ainsi que l'instabilité des prix agricoles et de la pauvreté, les ménages ruraux sénégalais sont contraint de trouver des moyens de combler le déficit de revenus agricoles induit par le contexte par la pratique d'activités non agricoles via notamment les services et les petites industries rurales. Ce processus s'inscrit dans le contexte d'une transformation rurale bien qu'évoluant lentement en raison de certains indicateurs telle que la part croissante des agriculteurs en milieu rural. Ces derniers représentent 73,8\% de ménages agricoles en milieu rural selon le rapport définitif 2013 du Recensement général de la population et de l'habitat, de l'agriculture et de l'élevage ; un rapport produit par l'Agence Nationale de la Statistique et de la Démographie du Sénégal (ANSD). Dans une telle situation où l'économie est fondée sur l'agriculture (cas pour la plupart des économies d'Afrique subsaharienne), la stagnation de la demande effective constatée, découle de la faiblesse des revenus et de l'absence d'infrastructures reliant les zones rurales aux petites et grandes villes.

Les ménages agricoles ruraux sont confrontés à une insuffisance de ressources financières, puisque 
l'agriculture dont ils dépendent reste fortement tributaire de la pluviométrie qui demeure depuis un certain temps irrégulière et imprévisible. Or la diminution des revenus agricoles remet en cause l'espoir fondé sur l'agriculture comme source d'emploi et comme facteur de croissance, puisqu'un secteur qui n'est pas capable de générer des activités rémunératrices n'attirera pas les jeunes qui arrivent en masse sur le marché du travail (Dupaigre, 2017). Selon les projections de Dupaigre, neuf pays dont le Sénégal verraient les revenus de leurs agriculteurs décroitre de façon chronique en raison de l'effondrement de la productivité des terres. En conséquence, on pourrait s'attendre à l'accroissement des pressions migratoires du fait de l'accentuation de la pauvreté et la fréquence des crises de subsistance.

Selon la revue «Grain de Sel » (2012) et d'après les études Ruralstruct, une cohorte annuelle de 200000 à 300000 jeunes pour des pays d'environ 15 millions d'habitants comme le Sénégal, arrivent chaque année sur le marché du travail. Comment dans cette situation contraindre les populations à rester ou à revenir dans le secteur agricole? Quelle stratégie adopter dès lors pour relever le niveau de revenu de ces populations ? De même, quand le taux de croissance démographique atteint les $3 \%$ et que l'activité économique principale, l'agriculture en l'occurrence n'est pas suffisamment industrialisée autrement dit reste encore au stade d'agriculture familiale, comment ne pas penser à créer des emplois ?

Des transformations structurelles et rurales relativement rapides sont par conséquent nécessaires, notamment pour favoriser la croissance du secteur rural non agricole (industries et services) et resserrer les liens entre les villages et les zones péri-urbaines (Barrett et al. 2017). Selon Boto et al. (2011), l'agenda de cette transformation rurale vise à améliorer la qualité de vie globale dans les zones rurales.

A travers l'emploi ou l'entrepreneuriat direct, l'activité non-agricole ${ }^{1}$ peut être bénéfique aux pauvres et les autres ruraux résidents. Ainsi, augmenter les opportunités et les capacités du monde rural à bénéficier de l'économie rurale non-agricole est essentiel pour des perspectives d'élimination de la pauvreté et de l'augmentation de la prospérité (BM, 2015).

\section{Revue de la littérature}

\section{I.1. Définition et généralités sur la transformation rurale}

La transformation rurale est l'objet de nombreuses tentatives de définitions (cf. Berdegué, Rosada \& Bebbington 2011; Freguin-Gresh, White \& Losch 2012). Celle qui nous convainc davantage est la définition élaborée par le NEPAD (2013) parce que plus complète et précise. Pour cette institution, la transformation rurale demeure « un processus global de changement sociétal par lequel les sociétés rurales diversifient leurs économies et réduisent leur dépendance vis-à-vis de l'agriculture ». Parmi les impératifs majeurs définis par l'agenda de la transformation rurale figurent la création d'emplois en nombre suffisant et de qualité et l'autonomie économique des petites et moyennes villes rurales. La diversification économique rurale est un moteur clé de création d'emplois (Boto et al. 2011). Cette diversification implique ainsi la création de multiples services et industries rurales autrement dit, toute autre activité différente de celle qui est reconnue comme étant la principale activité économique du monde rural.

De 2000 à 2014, le PIB par habitant en Afrique subsaharienne a augmenté de près de 35\% en termes réels, doublant dans certains pays. Ces progrès ont été réalisés alors que la croissance de la productivité agricole est restée globalement faible. Pour ainsi mettre fin à l'extrême pauvreté, il faudra un changement structurel dans l'agriculture et, plus généralement, dans les économies rurales africaines (Barrett et al. 2017). La clef de cette transformation structurelle de l'économie rurale consiste à permettre aux producteurs ruraux de suivre fidèlement l'évolution de la demande à mesure que l'économie se développe et que les revenus augmentent. Cela signifie qu'il faut cesser de mettre uniquement l'accent sur l'augmentation de la productivité agricole et accorder une plus grande attention aux activités rurales non agricoles (CNUCED (a), 2015).

Cependant, pour cette institution, la grande majorité des PMA, toutes catégories confondues, n'en sont encore qu'au premier stade de la transformation de leur économie rurale, les activités rurales non agricoles étant toujours principalement axées sur l'agriculture (bien que souvent réparties presque à parts égales entre le commerce, l'industrie manufacturière et les autres services) et essentiellement exercées dans le secteur informel. Toutefois, les catégories de «pays à vocation agricole » et de «pays en mutation » utilisées comme indicateurs dans le Rapport sur le développement dans le monde 2008 de la Banque mondiale donnent à penser qu'un petit nombre de PMA africains et asiatiques à l'image de l'Angola, du Bangladesh, de l'Ouganda et du Sénégal, ont engagé la deuxième phase de cette transformation.

Un exemple de modèle à suivre est celui de la Grande-Bretagne, pionnière de l'industrialisation, et qui a amorcé la transformation d'une société agraire en une société industrielle au milieu du XVIIIe siècle. La plupart des pays d'Europe continentale ont emboîté le pas au cours du XIXe siècle. Compte tenu de l'impact de ce modèle sur le débat actuel sur la transformation rurale dans les économies à revenu faible ou intermédiaire, il semble raisonnable d'explorer dans quelle mesure ce modèle pourrait servir à éliminer la pauvreté dans ces pays

\footnotetext{
${ }^{1}$ Les activités non agricoles ou encore Economie rurale non agricole font référence aux industries et services ruraux.
} 
(Rauch et al. 2016).

Headey et Dorosh (2011), (cité par le NEPAD, 2013)) fournissent un certain nombre de facteurs clés de la transformation structurelle (rurale). Il s'agit d'abord d'une transformation des résultats se traduisant par une baisse dans la part de la valeur ajoutée créée dans le secteur agricole et une augmentation dans la part de la valeur ajoutée créée dans le secteur non- agricole (industrie, fabrication, mine, construction ou services). Il s'agit ensuite d'une transformation de l'emploi, c'est-à-dire une baisse dans la part d'heures travaillées par la main d'œuvre du secteur agricole et une augmentation dans la part des heures travaillées par la main d'œuvre du secteur non-agricole.

Cette notion de transformation rurale a été si importante qu'elle a attiré une plus grande attention dans le débat sur la politique universitaire et le développement suite à la flambée des prix agricoles en 2008 et à la renaissance du développement rural et agricole. Presque toutes les organisations internationales impliquées dans ce domaine ont commandé des études ou des notes conceptuelles sur le sujet (Rauch et al. 2016).

\section{I.2. Composition et caractéristiques des industries et services ruraux}

Selon l'Agence Nationale de la Statistique et de la Démographie (ANSD) du Sénégal, le secteur des industries est composé d'une multitude d'activités. Ce sont les branches manufacturières, l'extraction minière, les BTP, la transformation des produits agricoles ainsi que les métiers liés à la production et à la distribution de l'eau, de l'électricité et du gaz. Les services par contre sont composés du commerce, des services ruraux, de l'administration, l'hôtellerie et la restauration, le transport et les autres services.

Les industries locales et petits business sont selon Faruqee (ND) et Igwe (2013), génératrices d'emploi et d'innovation et peuvent en outre contribuer à la transformation structurelle. Ils forment d'après Mukherjee et al. (2005) une partie intégrante de la production et de l'emploi du secteur rural. Les revenus dérivés de telles activités sont de plus en plus importants dans plusieurs pays et fournissent souvent des liens déterminants pour la croissance de l'agriculture.

Des études récentes suggèrent en outre que les mouvements de main-d'œuvre de l'agriculture vers l'économie non agricole et les villes secondaires sont particulièrement efficaces pour réduire la pauvreté plus que les mouvements dans les grandes villes (Barrett et al. 2017). Selon Haggblade et al. (2009), dans les pays dont l'économie est en train de passer de l'agriculture à d'autres secteurs, les décideurs considèrent l'économie rurale non agricole comme un secteur capable d'absorber de manière productive les nombreux travailleurs agricoles et petits exploitants qui sont exclus de l'agriculture par des modes de production de plus en plus commerciaux et à forte intensité capitalistique. Compte tenu des besoins en capitaux souvent faibles dans l'économie non agricole, les décideurs considèrent que l'économie rurale non agricole offre un moyen de sortir de la pauvreté pour nombre de leurs pauvres en milieu rural. Cette capacité de l'économie non agricole à pouvoir réduire la pauvreté serait due à ses faibles besoins en capitaux.

Parmi les activités impliquées, les services sont les plus importants en termes de sources de revenus. Le secteur des services en Afrique représente la moitié de la production du continent et il est devenu prépondérant dans un certain nombre de pays africains. La croissance des services a été extrêmement dynamique en Afrique avec un taux deux fois plus supérieur au taux moyen dans le monde sur la période 2009-2012. Leur dynamique a assurément contribué à la croissance qu'a connue l'Afrique au cours de la dernière décennie. Cependant, dans les pays où il occupe une place importante, les services ne représentent pas nécessairement une large part de l'emploi formel et que certains secteurs à très forte croissance dans les pays d'Afrique de l'Ouest (Commerce de gros et de détail, restauration et transport) sont dominés par des entreprises informelles (CNUCED b, 2015).

Le secteur des services doit soutenir le processus de transformation structurelle, c'est à dire le passage d'activités à faible productivité à des activités à forte productivité, et une diminution de la part de l'agriculture dans la production et l'emploi, allant de pair avec un accroissement de la part des activités manufacturières et des services modernes dans la production (CNUCED b, 2015).

Puisque l'agriculture à elle seule ne peut réduire la pauvreté rurale, il est nécessaire selon Boto et al. (2011) de promouvoir des entreprises non agricoles durables pour une création davantage d'emplois de meilleure qualité. Il est également nécessaire de souligner que les parts du revenu de l'emploi pouvant être dérivées de ces deux secteurs non agricoles à savoir les industries et les services sont comparables d'un pays à l'autre ayant un niveau de développement similaire. Cela souligne la nécessité d'accorder une plus grande attention à la croissance des entreprises non agricoles formelles dans les zones rurales et les petites villes de l'ASS (Barret et al. 2007).

\section{Sources de données}

Les données utilisées proviennent de l'Enquête de Suivi de la Pauvreté au Sénégal (ESPS), effectuée en 2011. Cette enquête s'articule essentiellement autour du renseignement des indicateurs de suivi des conditions de vie et la pauvreté. Elle s'est déroulée dans tout le territoire national. 


\section{Méthodologie}

III.1. Cadre théorique

L'hypothèse formulée pour cet article est celle que les revenus non agricoles (Industrie, Services, Commerce, Autres branches) sont supérieurs aux revenus agricoles. L'idée derrière cette hypothèse est que si ces revenus non agricoles sont plus conséquents que ceux provenant de l'agriculture, il y a nécessité d'inciter les ménages ruraux les plus vulnérables ${ }^{1}$ à savoir les pauvres sans terre ou avec juste de petites parcelles, d'entreprendre l'une de ces sources de revenus non agricoles comme principal emploi.

Pour vérifier cette hypothèse, nous avons effectué des estimations à l'aide du modèle logit ordonné. Comme son nom l'indique, le modèle logit ordonné permet d'ordonner les différentes catégories de revenu de la variable dépendante. Autrement dit, de les catégoriser du revenu le plus faible à celui le plus élevé.

Après avoir essayé nos estimations avec le modèle logit simple avec des revenus à l'état brut (non encore classifiés), on s'est rendu compte que le logiciel Stata ne permet pas d'afficher correctement l'ensemble des estimations (résultats). Il nécessitait alors de trouver une alternative à ce modèle logit simple. On a ainsi utilisé le modèle logit ordonné qui consiste à classer les revenus par catégories.

Pour éviter cependant une classification arbitraire de ces revenus avec le modèle alternatif, nous les avons catégorisés en quartile de revenu, classifiés et renommés de la manière suivante :

0 - Premier quartile $=$ Revenus très faibles

1- Deuxième quartile $=$ Revenus faibles

2- $\quad$ Troisième quartile $=$ Revenus moyens

3- Quatrième quartile $=$ Revenus élevés.

Les revenus ayant ainsi servi à faire cette comparaison sont ceux des chefs de ménages puisque d'autres sources de revenus existent évidemment dans les ménages.

Nous avons ainsi supposé que ces chefs de ménages ont plus le réflexe de pratiquer une autre activité (par exemple un service ou une activité industrielle en parallèle ou en remplacement à l'agriculture), puisque c'est à ces chefs de ménages que revient souvent la presque totalité de la charge familiale en zone rurale. Ce qui signifie que, ce sont uniquement les différents revenus des chefs de ménage, soient-ils des agriculteurs ou pratiquants d'autres activités relatives aux secteurs des industries et des services, qui sont comparés. Les autres sources de revenus des ménages ne sont pas prises en compte.

La branche d'activité principale constitue la variable explicative de notre modèle. Elle est entre autres le type d'activité (agriculture ou non) qu'exerce le chef de ménage et dont les revenus sont soumis à comparaison. Elle est composée de cinq types d'activité à savoir l'agriculture, l'industrie, le commerce, les services et les autres branches marchandes (autres services).

La variable de référence choisie par le logiciel stata parmi les variables explicatives est la variable «agriculture », puisque cette activité occupe plus d'exerçants que les autres secteurs d'activité. Les autres variables, à savoir les services, l'industrie, le commerce et les autres services seront ainsi comparées à la variable «agriculture ». Les résultats obtenus nous permettront d'aboutir à une confirmation ou une infirmation de l'hypothèse.

\section{III.2. Le Modèle d'Estimation}

La spécification mathématique du modèle se traduit par l'équation suivante :

$\mathrm{P}(\mathrm{Yi}>\mathrm{j})=\frac{\operatorname{Exp}(\alpha j+\mathrm{Xi} \beta)}{1+\{\operatorname{Exp}(\alpha j+\mathrm{Xi} \beta)\}}$

Avec $\mathrm{j}=1,2, \ldots, \mathrm{M}-1$

$\mathrm{Y}=$ Revenu $=$ Variable dépendante

$\mathrm{X}=$ Branche d'activité principale $=$ Série des Variables explicatives

$\mathrm{M}=$ Nombre de catégories de la variable dépendante ordinale

$\mathrm{J}=$ Nombre de modalités de la variable de la variable explicative

Ce qui implique que :

$\mathrm{P}\left(\right.$ Revenu $\left._{\mathrm{i}}>\mathrm{j}\right)=\frac{\operatorname{Exp}(\alpha j+\mathrm{Bracti} \beta)}{1+\{\operatorname{Exp}(\alpha j+\mathrm{Bracti} \beta)\}}$

En appliquant les variables au modèle, nous obtenons l'équation suivante :

Revenu $=\beta_{\mathrm{i}}$ Branche d'activité principale (1)

Avec : $\beta_{\mathrm{i}}=$ (Coefficient) Paramètre à estimer ;

La branche d'activité principale comprend les activités suivantes \{Agriculture, Industrie, Commerce, Service, autres branches\}

Ce qui aboutit à l'équation suivante :

\footnotetext{
${ }^{1}$ Le sens voulu pour le terme « vulnérable » est l'exposition au risque de pauvreté, de décapitalisation.
} 
Revenu $_{\mathrm{i}}=\beta_{0}+\beta_{1}$ Agriculture $+\beta_{2}$ Industrie $+\beta_{3}$ Commerce $+\beta_{4}$ Services $+\beta_{5}$ autres $+\varepsilon_{\mathrm{i}}$ (2)

III.3. Statistiques descriptives des variables explicatives

Les statistiques descriptives montrent dans le Tableau 1 que l'activité principale à savoir l'agriculture au sens strict occupe $68 \%$ des chefs de ménages ruraux, suivi du Commerce avec $11 \%$, des activités du secteur de l'Industrie avec 8\%, puis des Services avec 7\% et enfin des Autres Services avec 6\% des chefs de ménages. L'ensemble du secteur des services (à savoir les services, le commerce et les autres services) emploie ainsi près de $24 \%$ des actifs.

On s'aperçoit en outre dans le Tableau 2 que le commerce, les services, l'industrie et les autres services ont une part assez faible d'actifs et même nul pour certains cas concernant les catégories de Revenu très faible, faible, et moyen. Cela met en évidence toute l'importance des revenus provenant de telles activités.

\section{Tableau 1 : Statistiques descriptives des variables du modèle}

\section{Freq}

Branches d'activités (Percent)

Principales

\begin{tabular}{ll} 
Agriculture & 2584 \\
& $(67.93)$ \\
Industrie & 314 \\
& $(8.254)$ \\
Commerce & 411 \\
& $(10.80)$ \\
Services & 274 \\
& $(7.203)$ \\
autres Services & 221 \\
& $(5.810)$ \\
Total & 3804 \\
\hline
\end{tabular}

Tableau 2 : Statistiques descriptives des variables en fonction du Revenu

\begin{tabular}{|l|l|l|l|l|l|}
\hline Revenu & Très Faible & Faible & Moyen & Elevé & Total \\
\hline Branche d'activité & 32 & 37 & 141 & 2374 & 2584 \\
\hline Agriculture & 1 & 2 & 4 & 307 & 314 \\
\hline Industrie & 0 & 0 & 19 & 392 & 411 \\
\hline Commerce & 1 & 0 & 1 & 272 & 274 \\
\hline Services & 0 & 0 & 4 & 217 & 221 \\
\hline Autres Services & 34 & 39 & & 3562 & 3804 \\
\hline Total & & & & \\
\hline
\end{tabular}


Tableau 3: Résultats des estimations

\begin{tabular}{|c|c|c|}
\hline VARIABLES & $\begin{array}{l}\text { (1) } \\
\text { Logit coeff }\end{array}$ & $\begin{array}{l}(2) \\
\text { Odds ratio }\end{array}$ \\
\hline \multicolumn{3}{|c|}{$\begin{array}{l}\text { Revenu } \\
\text { Agriculture (Référence) }\end{array}$} \\
\hline Industrie & $\begin{array}{l}1.355 * * * \\
(0.389)\end{array}$ & $\begin{array}{l}3.877 * * * \\
(1.508)\end{array}$ \\
\hline Commerce & $\begin{array}{l}0.618 * * \\
(0.246)\end{array}$ & $\begin{array}{l}1.855^{* *} \\
(0.456)\end{array}$ \\
\hline Services & $\begin{array}{l}2.488 * * * \\
(0.713)\end{array}$ & $\begin{array}{l}12.03 * * * \\
(8.583)\end{array}$ \\
\hline Autres Services & $\begin{array}{l}1.576^{* * *} \\
(0.510)\end{array}$ & $\begin{array}{l}4.835 * * * \\
(2.464)\end{array}$ \\
\hline Constant cut 1 & $\begin{array}{l}-4.454 * * * \\
(0.174)\end{array}$ & $\begin{array}{l}0.0116 * * * \\
(0.00203)\end{array}$ \\
\hline Constant cut 2 & $\begin{array}{l}-3.677 * * * \\
(0.121)\end{array}$ & $\begin{array}{l}0.0253 * * * \\
(0.00307)\end{array}$ \\
\hline Constant cut 3 & $\begin{array}{l}-2.423 * * * \\
(0.0720)\end{array}$ & $\begin{array}{l}0.0887 * * * \\
(0.00638)\end{array}$ \\
\hline Observations & 3804 & 3804 \\
\hline
\end{tabular}

\section{III.4. Commentaire des résultats}

L'ensemble des interprétations sera basé sur les odds ratios. Les odds ratios encore appelés rapport des chances nous permettront dans ce travail d'exprimer le degré de dépendance entre les variables. Ainsi, les odds ratios des variables explicatives (Industrie, Commerce, Services, Autres Services) seront comparés à celui de l'agriculture qui est notamment la référence et équivaut à l'unité. L'agriculture est choisie comme référence par le logiciel Stata, mais aussi en réponse à la vérification de notre hypothèse de comparer les revenus des autres secteurs d'activité à celle-ci. Les résultats sont globalement significatifs au seuil de 1\% pour les industries, les services et les autres services et au seuil de 5\% pour le commerce.

III.4.1 Revenus des services

Dans nos estimations, on note que les chances relatives que le revenu d'un ménage exerçant une activité du secteur des services, sont de 11, 03 fois plus élevées que les revenus des ménages dont l'activité principale est l'agriculture. Ce qui signifie que tout accroissement des activités du secteur des services contribue à rendre plus probable une augmentation du revenu du ménage. Reardon (2006) dans son étude sur la diversification des revenus des ménages par les activités rurales non agricoles, confirme également l'importance des revenus provenant des services ruraux en soutenant que contrairement à la sagesse traditionnelle (reflété dans une longue histoire de programmes de gouvernement et de donneurs visant les industries rurales plutôt que les services) le revenu du secteur des services est souvent plus important que celui des industries rurales. Ceci témoigne tout l'intérêt à développer les activités relatives à ce secteur.

La CNUCED b (2015) suggère ainsi que vu l'importance des services ruraux dans le revenu des ménages, le secteur des services doit soutenir le processus de transformation structurelle. Celle-ci consiste au passage d'activités à faible productivité à des activités à fortes productivités et une diminution de la part de l'agriculture dans la production et l'emploi. Ceci va de pair avec un accroissement de la part des services modernes dans la production.

III.4.2 Revenus des industries rurales

En ce qui concerne le secteur des industries, les chances relatives que le revenu d'un ménage exerçant une activité industrielle, sont de 2,87 fois plus élevées que chez les ménages exerçant l'agriculture. Autrement dit tout accroissement des activités du secteur des industries contribue à rendre plus probable une augmentation du revenu du ménage. Il y a lieu de noter qu'en outre, beaucoup d'activités de diversification poursuivies par les ruraux impliquent les microentreprises, et l'importance des microentreprises dans la génération d'emplois et de revenus en zones rurales en Afrique est de plus en plus reconnue (Igwe, 2013). Elles peuvent, selon le FIDA (2004), offrir de nouvelles possibilités non négligeables de création de revenus pour les paysans sans terre et aussi être utiles à l'économie rurale, en créant de nouveaux moteurs de croissance économique et en contribuant à améliorer les conditions de vie locales. 


\section{III.4.3. Revenus du commerce}

Pour ce qui est du sous-secteur du commerce les chances relatives que le revenu d'un ménage exerçant une activité commerciale, sont de $85 \%$ plus élevées que chez les ménages agricoles. En d'autres termes, dans $85 \%$ des cas, les revenus provenant d'une activité commerciale quelle qu'elle soit, restent supérieurs à ceux provenant de l'activité principale qu'est l'agriculture. Le sous-secteur commerce a employé en 2011 dans la zone agro écologique la plus vaste du Sénégal en l'occurrence le Bassin arachidier plus de 9\% d'actifs et sa part en 2005 était de 17,32\% (Tandjigora, 2018). Cette baisse du nombre d'actifs a négativement impacté sur l'emploi des deux secteurs, l'industrie et les services. La part des actifs était passée de 28\% à 24\% entre 2005 et 2011 (Tandjigora, 2018). Cela montre que l'activité commerciale doit être plus valorisée. L'importance de la masse monétaire qu'il mobilise de même que son dynamisme et l'importance des emplois qu'il génère pourraient constituer une alternative à la crise de l'économie locale (PRDI Diourbel, 2008).

III.4.4. Revenus des autres branches d'activités

Les autres branches d'activités jouent également un rôle très important. Les exerçants de ces activités ont 3,83 fois plus de chances d'avoir plus de revenus que ceux qui exercent une activité agricole à titre principal. Cela signifie que, tout accroissement de ces types d'activités contribue à rendre plus probable une augmentation du revenu du ménage. Au-delà des services, ce sont les activités les plus appréciables en termes de création de revenus au profit des ménages. Il serait donc judicieux de multiplier ces activités afin que les revenus varient à la hausse et que les ménages puissent s'écarter de la pauvreté.

\section{Conclusion et recommandations}

Longtemps assimilée à la transformation agricole à travers l'étude des systèmes et chaines de valeur agricoles, la transformation rurale a depuis quelques décennies, bénéficié d'études nouvelles prenant suffisamment en compte les activités non agricoles. La perception de ces activités non agricoles n’est cependant pas unanime. Ainsi, les chercheurs et certains spécialistes des questions économiques non agricoles ont le plus souvent fait une analyse globale de cette partie de l'économie rurale. De nos constatations, ils ne procèdent pas d'habitude à une identification des différentes activités exercées par les ménages mais plutôt à un examen restreint des occupations de ceux-ci. Ils se limitent dans la plupart de leurs études aux notions d'emplois salariés et d'auto emplois sans toutefois préciser avec des données statistiques les composantes de ces groupes d'emploi. Dans cet article, les activités non agricoles ont été classifiées d'une manière sectorielle à savoir le secteur des industries et celui des services. Cela permet d'avoir un réel aperçu avec des données statistiques sur les types d'emploi qu'exercent les ménages, la fréquence dans l'occupation, et de pouvoir faire une étude comparative entre les revenus provenant de ces secteurs d'activité et l'agriculture.

L'hypothèse que l'on avait émise au départ, selon laquelle les revenus provenant des industries et services ruraux sont supérieurs à ceux provenant de l'agriculture, a été validée. L'objectif est d'inciter les ménages ruraux à s'intéresser davantage aux activités non-agricoles puisqu'elles génèrent plus de revenus. En réalité, là où l'agriculture rapporte 1 franc à son pratiquant, le service au même moment lui procure 12 francs, l'industrie 3,87 francs, le commerce 1,85 francs contre 4,83 francs pour les autres services, soit près de 4 Francs d'écart de revenus avec l'agriculture, ce qui représente une différence non négligeable. Les industries et services ruraux constituent à cet effet une réelle alternative à l'agriculture constamment confrontée aux réalités environnementales greffées au décor de la ville. Toutefois l'agriculture continue d'occuper la majorité des ruraux au Sénégal à l'instar de plusieurs pays d'Afrique subsaharienne, même si la plupart d'entre eux sont pauvres.

Le fait que l'agriculture continue d'employer la majorité des actifs ruraux même avec des revenus non conséquents au détriment des services et industries rurales se traduit par une contradiction évidente. La réalité suggérerait que les actifs devraient être plus attirés par des sources de revenus plus importantes. Notre article a apporté des preuves évidentes à cet effet. Il s'avère nécessaire de préciser en amont que parmi les activités non agricoles qui ont fait l'objet d'analyse dans cet article, les services sont mieux rémunérés que les petites industries rurales même s'ils sont exercés de manière informelle. Le secteur des services, comme le suggère la CNUCED en 2015, peut contribuer à la transformation structurelle, à la croissance économique et au développement en Afrique si des efforts concertés sont accomplis pour l'intégrer dans l'économie formelle si davantage d'emplois formels sont créés et des stratégies mises en œuvre en vue du développement. Le revenu non-agricole soutenu est susceptible de favoriser de nouveaux investissements dans l'économie agricole et de se substituer par conséquent aux marchés financiers manquants ou imparfaits. Ces deux secteurs (industrie et services) nécessiteraient d'être accompagnés dans leur processus de massification; ils mériteraient tout aussi fort l'appui des politiques publiques.

\section{Références bibliographiques :}

ANSD (2013) "Rapport définitif du Recensement général de la population et de l'habitat, de l'agriculture et de l'élevage", Agence Nationale de la Statistique et de la Démographie du Sénégal, 2013.

Barrett, C., Christiaensen, L., Sheahan, M. and Shimeles, A. (2017), "On the Structural Transformation of Rural 
Africa, Journal of African Economies", Vol. 26, AERC Supplement 1, i11-i35.

Berdegué, J.A., Rosada, T. and Bebbington, A.J. (2012), "Rural transformation”, Book chapter (27), 44 Pages, DOI:10.1093/acprof:oso/9780199671656.003.0028

Boto, I., Fotabong, E., Proctor, F., Lopes, I. and Kebe, H. (2011), Principaux facteurs de transformation rurale en Afrique. Ressources sur Transformation rurale en Afrique, Briefing n² 24 . CTA, NEPAD.

CNUCED (a) (2015), Rapport 2015 sur les pays les moins avancés. Transformer l'économie rurale. Aperçu général.

CNUCED (b) (2015), "Le développement économique en Afrique, Libérer le potentiel du commerce des services en Afrique pour la croissance et le développement". Rapport 2015

Dupaigre, B. (2017), "Revenus et Trajectoires agricoles en Afrique d'ici 2050: Vers un trop plein d'agriculteurs?", Notes techniques n²6, Mars 2017, AFD.

Faruqee R. (ND), "Rural Economy: Driver of Growth and Poverty Alleviation", Review of Cross-country, Experiences.

FIDA (2004), La politique du FIDA relative aux entreprises rurales.

Freguin-Gresh, S., White, E. and Losch B. (2012), "Rural Transformation and Structural Change: insights from Developing Countries facing Globalization", International Farming Systems Association 2012 Symposium, Workshop 1.3: Understanding agricultural structural changes and their impacts to support inclusive policy dialogue and formulation. Session: Toward a better understanding of agricultural transformations.

Grain de Sel, (2012), “Quelles politiques pour les populations rurales pauvres du Sahel ?" La revue d'interréseaux, Développement rural, n ${ }^{\circ}$ 59-62 - juillet 2012 - juin 2013.

Haggblade, S., Reardon, T. and Hazell, P. (2009), "Transforming the Rural Non-farm Economy. Opportunities and Threats in the Developing World”. IFPRI Issue Brief 58 February 2009.

Headey, D. D. and Dorosh, P. (2011), "The theory and empirics of Africa economic transformation: How far do they take us?" Paper presented at the IFPRI-University of Ghana Conference "Understanding Economic Transformation in Sub-Saharan Africa" May 10-11, 2011. In draft.

Igwe, P. (2013), "Rural Non-farm Livelihood Diversification and Poverty Reduction in Nigeria". Doctoral Thesis. School of Management (Plymouth Business School), University of Plymouth, Plymouth, PL4 8AA, United Kingdom, June 2013.

Mukherjee, A. and Zhang, X. (2005), "Rural Nonfarm Development in China and India: The role of Policies and Institutions". Discussion Paper n`24. IFPRI, Development strategy and governance division.

NEPAD (2013), "Transformation Rurale en Afrique : Un cadre pour orienter le Programme Rural Futures (Futurs Ruraux) du NEPAD”. Agence de Planification et de Coordination du NEPAD.

PRDI Diourbel (2008), "Plan Régional de Développement Intégré", Rapport final, horizon 2008-2012, Rapport bilan diagnostic.

Rauch, T., Beckmann, G., Neubert, S. and Rettberg, S. (2016), "Rural Transformation in Sub-Saharan Africa: Conceptual Study". SLE Discussion paper 01/2016 - EN. Centre for Rural Development (SLE) Berlin.

Reardon, T. (2006), "Household Income Diversification into Rural Nonfarm Activities". Forthcoming in Steven Haggblade, Peter Hazell and Thomas Reardon, editors. Transforming the Rural Nonfarm Economy (Baltimore: Johns Hopkins University Press, 2006).

Tandjigora, A. (2018), La Gestion du risque agricole par les activités non agricoles : Evidences de la zone rurale du Bassin arachidier du Sénégal, Thèse de Doctorat, Université Cheikh Anta Diop de Dakar, (à venir).

WB (2015), "Growing the Rural Non-Farm Economy to Alleviate Poverty: An IEG Evaluation of World Bank Group Support 2004-2014”, Approach Paper. December 8, 2015.

WB (2008), "Rapport sur le développement dans le monde, 2008, Banque mondiale. L'agriculture au service du développement. Résumé et commentaires", Mondes en développement 2008/3 (n 143), p. 117-136. DOI $10.3917 /$ med.143.0117. 